\title{
GAIA Fetal Growth Restriction Level of Diagnostic Certainty
}

National Cancer Institute

\section{Source}

National Cancer Institute. GAIA Fetal Growth Restriction Level of Diagnostic Certainty.

NCl Thesaurus. Code C128672.

A classification of maternal and fetal outcomes relating to fetal growth restriction, developed by the Global Alignment of Immunization safety Assessment in pregnancy, based on the extent to which the diagnosis has been confirmed. 